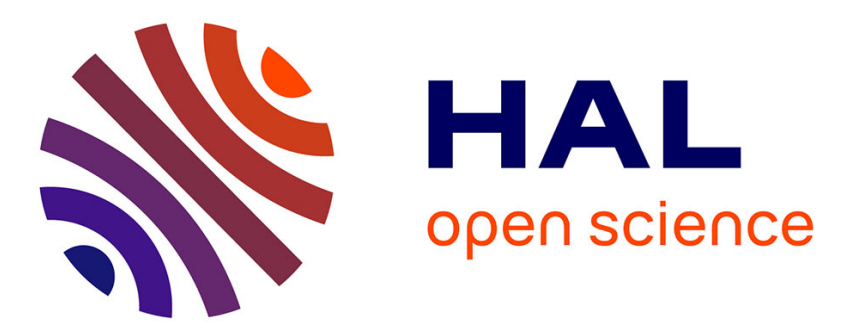

\title{
Parietomotor connectivity in the contralesional hemisphere after stroke: A paired-pulse TMS study
}

Etienne Allart, Arnaud Delval, Alexandre Caux-Dedeystere, Julien Labreuche, Romain Viard, Renaud Lopes, H. Devanne

\section{- To cite this version:}

Etienne Allart, Arnaud Delval, Alexandre Caux-Dedeystere, Julien Labreuche, Romain Viard, et al.. Parietomotor connectivity in the contralesional hemisphere after stroke: A paired-pulse TMS study. Clinical Neurophysiology, 2017, 128 (5), pp.707-715. 10.1016/j.clinph.2017.02.016 . hal-03183865

\section{HAL Id: hal-03183865 https://hal.science/hal-03183865}

Submitted on 29 Mar 2021

HAL is a multi-disciplinary open access archive for the deposit and dissemination of scientific research documents, whether they are published or not. The documents may come from teaching and research institutions in France or abroad, or from public or private research centers.
L'archive ouverte pluridisciplinaire HAL, est destinée au dépôt et à la diffusion de documents scientifiques de niveau recherche, publiés ou non, émanant des établissements d'enseignement et de recherche français ou étrangers, des laboratoires publics ou privés. 
Parietomotor connectivity in the contralesional hemisphere after stroke: a paired-pulse TMS study

Etienne Allart ${ }^{\mathrm{a}, \mathrm{b}}$, Arnaud Delval ${ }^{\mathrm{b}, \mathrm{c}}$, Alexandre Caux-Dedeystere ${ }^{\mathrm{d}}$, Julien Labreuche ${ }^{\mathrm{e}}$, Romain Viard ${ }^{b, f}$, Renaud Lopes ${ }^{b, f}$, Hervé Devanne ${ }^{\mathrm{c}, \mathrm{d}}$

${ }^{\text {a }}$ Neurorehabilitation Unit, Lille University Medical Center, F-59000 Lille, France

${ }^{\mathrm{b}}$ Univ. Lille, Inserm U1171-Degenerative and vascular cognitive disorders, F-59000 Lille, France

${ }^{\mathrm{c}}$ Department of Clinical Neurophysiology, Lille University Medical Center, F-59000 Lille, France

${ }^{\mathrm{d}}$ ULCO, URePSSS Unité de Recherche Pluridisciplinaire Sport Santé Société (EA7369), F-62228 Calais, France

${ }^{\text {e }}$ Department of Biostatistics, EA2694--Santé publique: épidémiologie et qualité des soins, Lille University, F59000 Lille, France

${ }^{\mathrm{f}}$ Clinical Imaging Core faCility, Lille University Medical Center, F-59000 Lille, France

\section{Corresponding author:}

Dr. Etienne Allart, MD

Neurorehabilitation unit

Hôpital Swynghedauw, CHRU de Lille

Rue André Verhaeghe

F-59037 Lille cedex, France

Tel: +33-320-444871; Fax: +33-320-445832.

Etienne.allart@chru-lille.fr

\section{Manuscript characteristics}

Word count: (references included)

References:

Figures:

Table:

\section{Conflicts of interest: none}

Acknowledgment: the authors thank David Fraser for editorial support.

Funding: this work was funded by the 2013 "hospital fund to assist emergence and structuring of activities and research teams" from the Lille University Medical Center, Lille, France; and the Rehabilitation Center of the Lille University Medical Center. 


\section{Highlights}

- PPC-M1 connectivity from posterior part of the PPC is disrupted in patients with spatial neglect

- aIPS-M1 connectivity in the contralesional hemisphere is unaffected in stroke patients

- The severity of motor impairment is not correlated with contralesional PPC-M1 connectivity

\section{Abbreviations:}

PPC: posterior parietal cortex

PMC: premotor cortex

M1: motor cortex

aIPS: anterior part of the intraparietal sulcus

pIPS: posterior part of the intraparietal sulcus

SPOC: superior parieto-occipital cortex

TMS: transcranial magnetic stimulation

ppTMS: paired-pulse TMS

CS: conditioning stimulus

TS: test stimulus

ISI: interstimulus interval

FDI: first dorsal interosseous

MEP: motor evoked potential

RMT: resting motor threshold

$\mathrm{N}+$ : neglect patients

$\mathrm{N}-$ : non-neglect patients

FMA-UE: upper extremity subsection of the Fugl-Meyer Assessment

MAL-14: 14-item Motor Activity Log questionnaire 


\begin{abstract}
Objectives: To assess the contralesional connectivity between the posterior parietal cortex (PPC) and the motor cortex (M1) in stroke patients, and to probe putative relationships with spatial neglect and motor impairment.
\end{abstract}

Methods: In 12 right-side stroke patients and 12 age-matched healthy controls, we used paired-pulse transcranial magnetic stimulation to assess the contralesional connectivity between three left-side PPC sites (the anterior intraparietal sulcus (aIPS), the posterior intraparietal sulcus and the superior parietooccipital cortex (SPOC)) and M1. The interstimulus interval (ISI) was set to 4 or $6 \mathrm{~ms}$.

Results: Although there were no differences between the stroke patient group and the controls, a subgroup analysis showed that stimulation over the SPOC with an ISI of $6 \mathrm{~ms}$ facilitated motorevoked potential responses in patients with neglect (and especially those with severe peripersonal neglect), relative to non-neglect patients. With an ISI of $4 \mathrm{~ms}$, the aIPS exerted an inhibitory influence on M1 in all subjects. The severity of motor impairment was not associated with PPC-M1 connectivity.

Conclusions: aIPS-M1 connectivity seems to be unaffected in stroke patients, whereas connectivity from the most posterior parts of the parietal cortex depends on the patient's neglect status.

Significance: These results provide insight into post-stroke changes in contralesional PPC-M1 connectivity.

Keywords: stroke; parietal cortex; transcranial magnetic stimulation; connectivity; spatial neglect; motor impairment 


\section{Introduction}

Stroke is the main cause of acquired disability in adults. Upper limb paresis is one of the most frequent symptoms after stroke; the recovery of upper limb function is often problematic but constitutes a key factor in personal autonomy. Spatial neglect (defined as a failure to acknowledge or explore stimuli towards the contralesional side) (Heilman et al. 2000) is another challenging consequence of stroke, since it slows recovery and impairs activities of daily living (Di Monaco et al. 2011). In fact, spatial neglect is a frequent consequence of lesions in the right hemisphere - especially those affecting the inferior parietal, temporoparietal and superior parietal territories (Mort et al. 2003; Verdon et al. 2010; Molenberghs et al. 2012; Rousseaux et al. 2013). As a sensory-motor interface and a key structure in movement planning and control, the posterior parietal cortex (PPC) may play a role in post-stroke cerebral plasticity and recovery from both upper limb paresis and spatial neglect (Buneo and Andersen 2006),

Parietofrontal networks (comprising the PPC, the premotor cortex (PMC) and the primary motor cortex (M1)) are significantly involved in the planning and online control of visually guided movements (Buneo and Andersen 2006; Filimon 2010; Davare et al. 2011; Vesia et al. 2013). More specifically, the most anterior structures of the PPC (i.e. the anterior part of the intraparietal sulcus (aIPS) and the supramarginal gyrus) are connected to the ventral PMC and control the grasping phase of movements (Davare et al. 2007; Koch et al. 2007, 2008a; Cavina-Pratesi et al. 2010; Koch et al. 2010; Vesia et al. 2013). In contrast, the most posterior structures in the PPC (i.e. the posterior part of the intraparietal sulcus (pIPS) and the adjacent cortical structures in the superior and inferior parietal lobule (the superior parieto-occipital cortex (SPOC) and the angular gyrus) are connected to the dorsal PMC and control the reaching phase (Makris et al. 2005; Koch et al. 2007, 2008a; Busan et al. 2009; Cavina-Pratesi et al. 2010; Karabanov et al. 2013; Vesia et al. 2013). Most of these findings on PPCM1 functional connectivity come from paired-pulse transcranial magnetic stimulation (ppTMS) studies (for a review, see (Rothwell 2011). At rest or just before movement initiation, a test stimulus (TS) is delivered to M1 a few milliseconds after a conditioning stimulus (CS) has been delivered to the PPC. Various studies of PPC-M1 connectivity in the left hemisphere have been performed in righthanded, healthy controls at rest. Depending on the intensity of CS and the length of the interstimulus interval (ISI, between the CS and the TS), one can variously observe an inhibitory influence of the CS when the latter is applied over the aIPS with an ISI of 4 ms (Koch et al. 2007; Karabanov et al. 2013; Vesia et al. 2013), a potentiating influence when the CS was applied over the pIPS (Koch et al. 2007; Karabanov et al. 2013), and the lack of an effect when the CS was applied over the SPOC (Vesia et al. 2013). However, SPOC-M1 connectivity may become functional during arm movements toward a target (Vesia et al, 2013).

Furthermore, transcallosal interparietal functional connections are asymmetric in right-handed 
healthy controls; the right PPC inhibits contralateral parietofrontal connections more strongly than the left PPC does (Koch et al. 2011). As a consequence, post-stroke neglect might result from the loss of interhemispheric balance between the right and left PPCs; this hypothesis is supported by the results of imaging studies in stroke patients (Rode et al. 2010; Bozzali et al. 2012; Lunven et al. 2015) and ppTMS studies in which contralesional (left) PPC-M1 connectivity was greater in right-handed patients with left spatial neglect than in patients without neglect or in healthy controls (Koch et al. 2008b, 2012). Subsequently, the application of repetitive TMS to the left PPC gave encouraging results in terms of recovery from peripersonal and behavioral neglect (for a review, see (JacquinCourtois 2015). In these ppTMS studies, however, the CS was applied over the pIPS with a broad figure-of-eight coil; this probably activated posterior structures in the superior and inferior parietal lobules. Finally, a growing body of literature data suggests that parietofrontal networks are involved in post-stroke recovery from motor impairment (Inman et al. 2012; Schulz et al. 2015, 2016).

In summary, the PPC and the associated parietomotor networks are key structures not only for gesture planning and control but also for spatial representations. Parietofrontal networks seems to be involved in the genesis of spatial neglect and may be related to motor recovery - making the PPC a possible target for therapeutic modulation by non-invasive brain stimulation techniques. However, functional specialization has been evidenced for subregions of the PPC in humans, and little is known about this aspect in stroke patients. Indeed, to the best of our knowledge, contralesional connections between subregions of the PPC (the aIPS, pIPS and SPOC) and M1 have not previously been studied in stroke patients.

Hence, the primary objective of the present study was to compare left PPC-M1 connectivity in right hemispheric stroke patients and in healthy controls by using a ppTMS protocol to assess the effect of a CS (applied over the aIPS, pIPS and SPOC) on M1 excitability. The secondary objective was to assess the relationship between spatial neglect and motor impairment on one hand and left (contralesional) PPC-M1 connectivity on the other.

\section{Methods}

\subsection{Study participants}

Patients were recruited from among in- and outpatients in the Neurorehabilitation Unit at Lille University Medical Center (Lille, France) between August 2014 and February 2016. We included 12 stroke patients who had suffered a single right ischemic or hemorrhagic hemispheric stroke (diagnosed by MRI) and had displayed left hemiparesis for at least 6 months before inclusion. The presence of spatial neglect was not an inclusion criterion but was assessed in all included patients (see 2.5). We also included 12 healthy age-matched healthy controls. We excluded patients with bilateral lesions, those who were unable to consent to or understand the study protocol due to language, cognitive or 
psychiatric disorders, and those presenting contraindications to TMS or MRI. All participants were right-handed, according to the Edinburgh Handedness Inventory (Oldfield 1971), and gave their informed written consent to participation. The study was approved by the local investigational review board (Comité de protection des personnes Nord Ouest IV, Lille, France; reference 2013-A01766-39), and was conducted in accordance with the tenets of the Declaration of Helsinki.

\subsection{Experimental procedures}

The participants were seated in a comfortable chair, with their right forearm resting on a second chair (the height of which could be adjusted). They were told to keep their arm still throughout the experimental session. As the first dorsal interosseous (FDI) muscle is activated during both phases of prehension movements (Koch et al. 2008a; Davare et al. 2009), motor evoked potentials (MEPs) were recorded at this site. Electromyographic (EMG) activity was recorded with $\mathrm{Ag}-\mathrm{AgCl}$ surface electrodes in a belly-tendon montage. The electrodes were positioned over the body of the muscle and the first metacarpophalangeal joint. A ground electrode was attached to the wrist. The EMG signals were amplified $(\times 1000)$, high-pass filtered at $10 \mathrm{~Hz}$, low-pass filtered at $1000 \mathrm{~Hz}$ (Digitimer, Hertfordshire, UK) and then sampling at $2 \mathrm{kHz}$ (1401 MicroMKII device; Cambridge Electronic Design, Cambridge, UK). Data were stored for subsequent off-line analysis using SIGNAL software (Cambridge Electronic Design, Cambridge, UK).

\section{$\underline{2.3 \text { Transcranial magnetic stimulation }}$}

We used a previously described ppTMS technique to study PPC-M1 interactions in the left hemisphere (for a review, see (Rothwell 2011). The CS was delivered at $90 \%$ of the resting motor threshold (RMT) using a figure-of-eight coil (PMD 25 decentral, Mag\&More, Münich, Germany; inner diameter 25mm) connected to a PowerMag100 stimulator (Mag\&More). The RMT was defined as the lowest possible stimulus intensity required to elicit MEPs with a $>50 \mu \mathrm{V}$ peak-to-peak in at least 5 out of 10 trials when participants kept the FDI relaxed in both hands (Rossini et al. 1994). The coil was held tangentially to the scalp surface and was turned slightly anticlockwise (by $15^{\circ}$ ) to induce a posterior-anterior-directed current in the underlying cortical tissue (Fig. 1a). The TS was delivered over M1 of the left hemisphere using a Magstim 200 stimulator (The Magstim Company Ltd, Whitland, UK), at an intensity that evoked MEPs with a $\sim 1 \mathrm{mV}$ peak-to-peak amplitude in the control condition. We placed a figure-of-eight coil (Magstim Alpha Branding Iron, The Magstim Company Ltd; external diameter: $50 \mathrm{~mm}$ ) tangentially to the scalp at an angle of $45^{\circ}$ to the mid-sagittal line, in order to induce a posterior-anterior-directed current across the central sulcus (Fig. 1a). The coils were held on to the scalp surface by articulated arms. The ISI was set to $4 \mathrm{~ms}$ or $6 \mathrm{~ms}$. At each site over the PPC, three conditions were tested in pseudorandom order: the TS alone (the control condition) and the combined CS-TS condition with the two ISIs. Eight responses were recorded for each condition, giving a total of 24 responses for each PPC site. The inter-trial interval was set to $5 \pm 1 \mathrm{~s}$. 


\subsection{Localization of brain sites}

The scalp location for M1 was defined as the point at which stimulation evoked the largest MEP in the right FDI. We used a stereotaxic neuronavigation system (eXimia ${ }^{\circledR}$ TMS, Nexstim, Helsinki, Finland) to accurately identify each PPC stimulation site and monitor the TMS coil position. Before the TMS session, participants underwent MRI on a 3T machine (Achieva, Philips Medical Imaging, Best, The Netherlands) equipped with an eight-channel head coil. Volumetric T1-weighted images were acquired with a magnetization-prepared gradient echo sequence (voxel size: $0.750 \mathrm{x}$ 0.727 x $0.727 \mathrm{~mm}^{3}$; repetition time $10.4 \mathrm{~ms}$; echo time $4.76 \mathrm{~ms}$; matrix size: 214 x 352 x 352 voxels). With regard to individual anatomical landmarks, we selected the three regions of interest within the PPC (the aIPS, pIPS and SPOC; Fig. 1b). The aIPS was defined as the region located over the intersection between the postcentral sulcus and intraparietal sulcus (Frey et al. 2005). The pIPS was situated over the intraparietal sulcus (Karabanov et al. 2013). The SPOC was defined as the region along the medial surface of the parietal lobe, anterior to the parieto-occipital sulcus, posterior to the subparietal sulcus and medial to the intraparietal sulcus (Vesia et al. 2013).

\subsection{Clinical assessment}

Patients were classified as suffering from spatial neglect ("N+") or not ("N-") with regard to their performance in the three tests described hereafter. Peripersonal neglect was first assessed using the bells test (Gauthier et al. 1989). In this test, participants have to identify 35 bell symbols distributed throughout a field of other symbols. Patients were considered to display peripersonal spatial neglect if they omitted at least 6 bells (Gauthier et al. 1989; Rousseaux et al. 2001). Personal (body) neglect was assessed with the fluff test (cut-off score: $\leq 13$ ), which consists in removing targets attached to the front of the participant's clothes (Cocchini et al. 2001). Lastly, behavioral neglect was assessed via the examiner's part of the Catherine Bergego Scale (cut-off score: > 10) (Azouvi et al. 2003).

Motor impairment was assessed via the upper extremity subsection of the Fugl-Meyer Assessment (FMA-UE), which comprises the motor function of the upper arm, wrist and hand, and overall coordination (Fugl-Meyer et al. 1975). The 14-item Motor Activity Log questionnaire (MAL14) was used to measure upper limb function in daily life; participants have to rate the amount and quality of movement of the paretic arm when completing 14 activities of daily living (Uswatte et al. 2005).

\section{$\underline{2.6 \text { Statistical analysis }}$}


Peak-to-peak MEP amplitude was analyzed as the raw data for each condition (TS and CS-TS at each ISI) and (after normalization) as a proportion of the MEP recorded under control conditions (delivery of the TS alone) during the same block of trials. The normality of the data distribution was checked with a Shapiro-Wilk test and Q-Q plots. Intergroup comparisons of demographic and strokerelated variables were performed using Student's t test (or the Mann-Whitney U test, for non-normally distributed data) for quantitative variables and Fisher's exact test for qualitative variables.

To study inter- and intragroup differences, we used linear mixed models for repeated measures (using an unstructured covariance pattern model, with restricted maximum likelihood approach as the estimation method) to take account of the correlation between repeated measures within the same subjects. For each linear mixed model, we checked the normality and homoscedasticity of the residuals.

To study intergroup differences in PPC-M1 connectivity, we first compared the normalized MEP amplitude at each PPC site in healthy controls vs. the whole stroke patient population (i.e. the study's primary objective). In these models, the dependent variable was the normalized MEP amplitude and the fixed effects were the group (healthy controls vs. patients), the ISI (4 ms vs. $6 \mathrm{~ms}$ ) and the group $\mathrm{x}$ ISI interaction. We next compared the normalized MEP amplitude at each PPC site among stroke patients (as a function of $\mathrm{N}+$ vs. $\mathrm{N}$ - status), using the same linear mixed model approach. In both analyses, post-hoc comparisons at each ISI were performed (using linear contrasts with Bonferroni correction) whenever the group x ISI interaction was statistically significant.

To study intragroup differences in PPC-M1 connectivity (i.e. to determine whether the MEP in the CS-TS conditions differed from the TS condition in each group: healthy controls, the whole stroke patient population, $\mathrm{N}+$ patients, and $\mathrm{N}$ - patients) for the three different PPC sites, we also computed separate linear mixed models in which the raw MEP amplitude was the dependent variable, and the condition (TS, and CS-TS at ISIs of 4 and $6 \mathrm{~ms}$ ) was the fixed effect. Whenever the effect of condition was significant, post-hoc comparisons of TS vs. each CS-TS condition were performed (using linear contrasts with Bonferroni correction).

Spearman's rank correlation coefficient was used to evaluate the respective associations between the severity of spatial neglect, upper limb function and motor impairment on one hand and contralesional PPC-M1 connectivity on the other.

The threshold for statistical significance was set to $\mathrm{p}<0.05$. All tests were two-tailed. All statistical analyses were performed with SAS software (version 9.3, SAS Institute, Cary, NC).

\section{Results}

\section{$\underline{3.1 \text { Patients }}$}

The characteristics of the study population are summarized in Table 1. Six of the 12 patients presented symptoms of spatial neglect. There were no significant differences between patients and 
healthy controls in terms of the mean age and the gender ratio. Even though $\mathrm{N}+$ patients had slightly higher FMA-UE scores and were slightly older than $\mathrm{N}$ - patients, the intergroup differences were not statistically significant (FMA-UE score: $\mathrm{p}=0.199$; age: $\mathrm{p}=0.108$; also disease duration: $\mathrm{p}=0.378$ ).

As seen on overlap maps (Fig. 2), the lesions were more widespread in the neglect group (mean \pm standard error of the mean $(\mathrm{SEM})$ volume $=134888 \pm 52085$ voxels in the $\mathrm{N}+$ group and $29444 \pm 25349$ in the $\mathrm{N}$ - group; $\mathrm{p}=0.015$ ) and were more likely to involve the parietal lobe. There was less lesion overlap in the $\mathrm{N}$ - group.

The ppTMS protocol was not performed for the aIPS in 2 patients (one in each group) and 4 healthy controls because this structure was too close to M1 (due to skull size) and so the coils could not be placed properly.

\subsection{The RMT and the M1 stimulation intensity}

The RMT for the left M1 (used to calibrate the intensity of the CS) was $65.3 \pm 12.7 \%$ of the maximum stimulator output in the patients $(70 \pm 14.5 \%$ in $\mathrm{N}+$ and $60.7 \pm 9.7 \%$ in $\mathrm{N}-)$ and $63.1 \pm$ $10.3 \%$ of the maximum stimulator output in healthy controls (Fig. 3a); the intergroup difference was not statistically significant. Likewise, there was no significant intergroup difference in the TS intensity needed to elicit a $1 \mathrm{mV}$ MEP in the control condition (Fig. 3b) or the MEP amplitude elicited in the control condition (TS) at any PPC site.

\subsection{Intergroup differences in PPC-M1 connectivity}

In the linear mixed models comparing all stroke patients with healthy controls, we did not observe any significant intergroup differences (group effect: $\mathrm{p}=0.53$; ISI $x$ group interaction: $\mathrm{p}=0.98$ ). Only a significant effect of the ISI on aIPS-M1 connectivity was observed ( $p=0.005)$ (Fig 4a). This indicated that MEP suppression was greater at an ISI of $4 \mathrm{~ms}$ than at $6 \mathrm{~ms}$ in all subjects, regardless of their group. Furthermore, we did not find any differences between the $\mathrm{N}+$ and $\mathrm{N}$ - groups (group effect: $\mathrm{p}=0.47$; ISI $\mathrm{x}$ group interaction: $\mathrm{p}=0.51$ ); only a non-significant difference between the two ISIs was observed $(\mathrm{p}=0.066)($ Fig $4 \mathrm{~b})$.

When comparing SPOC-M1 connectivity in the stroke patient population vs. healthy controls, the effects of group and ISI were not significant (group effect: $\mathrm{p}=0.51$; ISI effect: $\mathrm{p}=0.083$; ISI $\mathrm{x}$ group interaction: $\mathrm{p}=0.56$ ) (Fig 4e). Interestingly, when comparing $\mathrm{N}+$ and $\mathrm{N}$ - patients, the ISI $\mathrm{x}$ group interaction was significant $(\mathrm{p}=0.005)$. Post-hoc comparisons showed that when the CS was applied over the SPOC after an ISI of $6 \mathrm{~ms}, \mathrm{~N}+$ patients showed stronger facilitation than $\mathrm{N}$ - patients (Bonferroni-adjusted p=0.016) (Fig. 4f).

For pIPS-M1 connectivity, we did not find any group or ISI effects when comparing either the whole stroke patient population with healthy controls (group effect: $\mathrm{p}=0.15$; ISI effect: $\mathrm{p}=0.81$; ISI $\mathrm{x}$ group interaction: $\mathrm{p}=0.67$ ) or when comparing $\mathrm{N}+$ patients with $\mathrm{N}$ - patients (group effect: $\mathrm{p}=0.20$; ISI 
effect: $\mathrm{p}=0.65$; ISI $\mathrm{x}$ group interaction: $\mathrm{p}=0.55)$. $($ Fig $4 \mathrm{c}, \mathrm{d})$.

\subsection{Intragroup differences in PPC-M1 connectivity}

When comparing the non-normalized MEP amplitudes under the three conditions (TS alone vs. CS-TS at each ISI), a significant difference in aIPS-M1 connectivity was observed for healthy controls $(\mathrm{p}=0.05)$, the whole stroke patient population $(\mathrm{p}=0.028)$ and $\mathrm{N}+$ patients $(\mathrm{p}=0.015)$ but not for $\mathrm{N}$ - patients ( $\mathrm{p}=0.22$ ). Post-hoc comparisons revealed significant MEP suppression at an ISI of $4 \mathrm{~ms}$ for healthy controls, the whole stroke patient population, and the $\mathrm{N}+$ group (Bonferroni-adjusted $\mathrm{p}<0.05$, Fig 4a,b). A main effect of condition on SPOC-M1 connectivity was only found in N+ patients $(\mathrm{p}=0.028)$, in whom connectivity was significantly facilitated at an ISI of $6 \mathrm{~ms}$ (Bonferroni-adjusted $\mathrm{p}=0.037$ ) (Fig 4f). As with the intergroup comparisons, we did not find any difference between TS and CS-TS modalities after applying the CS over the pIPS ( $p>0.30$ for all comparisons) (Fig $4 c, d$ ).

\subsection{Relationships between PPC-M1 connectivity and clinical assessments}

As SPOC-M1 connectivity at an ISI of $6 \mathrm{~ms}$ discriminated between $\mathrm{N}+$ and $\mathrm{N}$ - patients, we chose this interval to study the association between the severity of neglect and PPC-M1 connectivity. Interestingly, patients who presented with more severe peripersonal neglect tended to show stronger MEP facilitation; indeed, the number of omissions in the bells test was significantly correlated with the degree of facilitation induced by PPC conditioning ( $\mathrm{r}=0.841 ; \mathrm{p}=0.036)$ (Fig 5). No such association was found for personal neglect (i.e. performance in the fluff test) and behavioral neglect (i.e. with the Catherine Bergego Scale).

Lastly, neither the degree of motor impairment (as assessed by the total FMA-UE score) nor upper limb function (as assessed by the MAL-14 score) was associated with contralesional PPC-M1 connectivity when analyzing either the whole patient group or the $\mathrm{N}+$ and $\mathrm{N}$ - subgroups separately. Likewise, the FMA-UE subscores for proximal motor function (volitional movements) and distal motor function (wrist and hand movements) were not correlated with PPC-M1 connectivity when the CS was applied to the posterior sites (the pIPS and the SPOC) and the anterior site (the aIPS), respectively.

\section{Discussion}

The present study is the first to have evaluated contralesional (left-side) PPC-M1 connectivity at rest in stroke patients by precisely targeting three subparts of the PPC, i.e. an anterior region (the aIPS) and two posterior regions (the SPOC and the pIPS). Although there were no intergroup differences when comparing the patients as a whole (regardless of their neglect status) with healthy controls, we demonstrated that applying CS over the SPOC at an ISI of $6 \mathrm{~ms}$ facilitated MEP 
responses in the $\mathrm{N}+$ group but not in the $\mathrm{N}$ - group or healthy controls - especially when peripersonal neglect was severe. Furthermore, ppTMS of the aIPS exerted an inhibitory influence on M1 in both patients and healthy controls. Lastly, the severity of motor impairment was not associated with PPCM1 connectivity.

\subsection{Functional connectivity between posterior regions of the PPC and M1}

Our results highlighted the fact that neglect status must be taken into account when assessing contralesional PPC-M1 connectivity at posterior PPC sites (the pIPS and the SPOC). Previous work has already shown that connectivity between the posterior part of the PPC and M1 is modified not only in the contralesional hemisphere of stroke patients with neglect (Koch et al. 2008b, 2012) but also in healthy controls (relative to a control condition) as a result of asymmetric interparietal functional connections (Koch et al. 2011). However, Koch et al. only studied the pIPS as the target for the CS. In the present study (and in contrast to previous works (Koch et al. 2008b, 2012)), we did not observe hyperexcitability in pIPS-M1 networks in either patients or healthy controls but did evidence hyperexcitability of parietomotor connections in the N+ group when the CS was applied over the SPOC after an ISI of $6 \mathrm{~ms}$. Koch et al. applied the CS over the pIPS (either located by the EEG coordinates or by neuronavigation) using a broad TMS coil; this would have modulated cortical regions over the angular gyrus and the posterior part of the superior parietal lobule. In the present study, we used a much smaller coil and thus produced a much narrower stimulation field. Furthermore, we placed the coil over the sulcus (Karabanov et al. 2013) and not over the superior part of the angular gyrus - in contrast to previous studies (Koch et al. 2008b, 2011, 2012). Accordingly, the regions bordering the sulcus (the angular gyrus and superior parietal lobule) would have been less modulated; this may be why we did not observe any effect when stimulating the pIPS. Moreover, the SPOC is close to the pIPS, and both regions are involved in planning and controlling visually-guided reaching movements - albeit with their own specific features (the encoding of reach goals for the SPOC, and reach vectors for the pIPS (Vesia et al. 2010)). Both regions produced the same facilitation pattern in ppTMS protocols when the CS was applied during movement planning (Koch et al. 2008a, 2010; Vesia et al. 2013). In a more integrated analysis, both the SPOC and the pIPS are posterior nodes in the dorsal attentional network. This network is known to mediate the top-down, guided, voluntary allocation of attention to location or features (Corbetta and Shulman 2002; Szczepanski et al. 2013; Vossel et al. 2014). Dysfunction to the dorsal attentional network - whether caused either directly (Ptak and Schnider 2010, 2011) or indirectly (by damage to the ventral attentional network; Corbetta and Shulman 2011) - is thought to be one of the mechanisms underlying spatial neglect. Finally, if lesions in the inferior parietal lobule or the superior temporal gyrus tend to lead to neglect whereas damage to the superior parietal lobule leads to optic ataxia (Vallar and Perani 1986), lesions to the IPS and superior parietal lobule lesions can be also involved in neglect (Molenberghs et al. 2012). Taken as a whole, these arguments strengthen the hypothesis whereby SPOC hyperexcitability 
in $\mathrm{N}+$ patients is related to neglect (as pIPS-M1 hyperexcitability was seen to be related to neglect in earlier studies).

Interestingly (and even though this result should be interpreted with caution, in view of the small number of patients with neglect), the amount of facilitation induced by the conditioning of SPOC at an ISI of $6 \mathrm{~ms}$ was correlated with the severity of peripersonal spatial neglect but not with personal or behavioral neglect. Koch et al. reported an association with peripersonal neglect when the CS was applied over the pIPS (Koch et al. 2008b), whereas fMRI studies have extended these links to behavioral neglect (Baldassarre et al. 2014; Ramsey et al. 2016). With regard to the specific function of the posterior regions of PPC, it would be interesting to study the motor component of neglect (directional hypokinesia), i.e. an impairment in initiating or completing movements in the leftward direction (Heilman et al. 1985; Kim et al. 2013). The lack of correlation between PPC-M1 connectivity and personal neglect is not surprising, as they represent two different entities that are sometimes but not always associated. Spatial attention is less involved in body perception, and the neuroanatomical correlates of personal neglect are different from those of peripersonal neglect (Baas et al. 2011; Rousseaux et al. 2015). Hence, in addition to the lesion site (as discussed above) and as already suggested in the lietrature (Koch et al. 2008b), the neglect subtype is another important factor to be taken into account when seeking to interpret abnormal PPC-M1 connectivity in patients with neglect. On the clinical level, the SPOC may be an alternative to the pIPS as a target for non-invasive brain stimulation protocols aimed at reducing left parietal hyperexcitability in patients with spatial neglect. Nevertheless, the results of the present pilot study must first be confirmed and extended.

\subsection{Functional connectivity between aIPS and M1}

Our data indicated that contralesional aIPS-M1 connectivity was maintained after stroke and was not influenced by spatial neglect. This result is not surprising, since anterior regions of the PPC are brought into play in the grasping phase of upper limb movements but are not involved in encoding and controlling reaching movements and thus the integration of the contralateral workspace. Furthermore, lesions in the anterior part of PPC are not a cause of neglect in stroke patients.

PPC-M1 connectivity was not correlated (whatever the target for the CS) with the severity of motor impairment and upper limb function. We hypothesize that contralesional PPC-M1 connectivity may be indirectly modified (via interhemispheric connections) in the lesioned hemisphere in paretic patients. Indeed, recent imaging studies have highlighted a potential association between resting state connectivity in the parietofrontal networks of the lesioned hemisphere (especially the connectivity from the aIPS) and residual motor function in stroke patients, together with the involvement of these networks in motor recovery (Inman et al. 2012; Schulz et al. 2015, 2016). In the future, the analysis of ipsilesional aIPS-M1 connectivity using a ppTMS protocol might be of greater relevance to the link between parietomotor connectivity and motor recovery. 


\subsection{Possible mechanisms underlying changes in PPC-M1 connectivity}

The most widely accepted explanation for contralesional PPC-M1 hyperexcitability in neglect patients is based on the notion of interhemispheric rivalry; the imbalance concerns homotopic regions of the two hemispheres, rather than the whole lesioned hemisphere. Unsurprisingly, we found that lesions of the parietal lobe were more frequent in $\mathrm{N}+$ patients, since these lesions often cause neglect and directly affect the interhemispheric balance. However, one must also bear in mind that remote lesions in the downstream structures of parietofrontal networks might also alter the PPC's interhemispheric balance - especially in cases of damage to the ventral attentional network (Corbetta and Shulman 2011; Lunven et al. 2015). The influence of the lesion site on the amount of interhemispheric imbalance (and thus on the hyperexcitability of PPC-M1 connectivity in the contralesional hemisphere) has not yet been explored. The small number of patients in the N+ group prevented us from performing this analysis in the present study. In the future, this question could be addressed by measuring PPC-M1 connectivity as a function of whether the neglect patient's lesion includes parietal areas. Moreover, ppTMS protocols can only study connections from parietal areas to motor areas; it might be interesting to use functional imaging or TMS-EEG to study the connectivity of other parietofrontal networks in general and direct connections to premotor areas (i.e. the ventral and dorsal PMC) in particular.

One can also speculate as to whether M1 excitability has a role in changes in PPC-M1 connectivity. As already reported in acute and chronic stroke patients, we found that the RMT for the contralesional hemisphere was similar when comparing the stroke group with healthy controls and when comparing $\mathrm{N}+$ patients with $\mathrm{N}$ - patients (Liepert et al. 2000; Manganotti et al. 2002); this indicated that PPC-M1 abnormalities in patients were probably not due to changes in the excitability of the corticomotoneural cells located in the primary motor cortex. Although the PPC neurons' mechanisms of action on M1 are poorly understood, it has been suggested that (i) there are direct, functional connections between several PPC regions and M1 (Koch et al, 2007) and (ii) MEP facilitation would arise from interaction with late I-wave circuits. Other researchers have shown that local inhibitory and excitatory interneurons may be dysfunctional in the contralesional hemisphere following stroke (Edwards et al, 2013; Mello et al, 2015). It is therefore conceivable that the changes in the posterior PPC's influence on M1 excitability observed after stroke could also be due to dysfunction in M1 interneuron circuits.

\subsection{Study limitations}

The present study suffered from several limitations. Firstly, the sample size was small (particularly in the N+ subgroup); this may have led to a lack of statistical power when studying the influence of spatial neglect and may possibly have biased the correlations. Secondly, it should be borne in mind that the number of LMMs was high, and so we cannot rule out false positives. Thirdly, the patient population was quite heterogeneous with regard to disease characteristics. To reduce this 
heterogeneity, we included only chronic stroke patients. However, this choice complicated the inclusion of patients with neglect because this impairment often disappears in the months following stroke. Moreover, despite our use of small coils, a lack of space prevented proper coil placement over the aIPS in 6 participants ( 2 stroke patients and 4 healthy controls); surprisingly, only one previous study has reported this type of problem (Karabanov et al. 2013). Lastly, we decided to study connectivity at 2 ISIs ( 4 and $6 \mathrm{~ms}$ ) only; this was done to reduce the numbers of comparisons and because PPC-M1 connectivity at these intervals was modified at rest in both stroke patients and healthy controls (depending on the PPC site). However, we cannot rule out the possible occurrence of PPC-M1 connectivity at longer ISIs. Even though these limitations emphasize that our present findings are hypothesis-generating and need to be replicated in larger studies, our present results open up new perspectives in the assessment of parietomotor connectivity after stroke.

\section{Conclusions}

Contralesional aIPS-M1 connectivity seems to be unaffected in stroke patients, whereas connectivity from the most caudal sites in the PPC was disrupted in patients with spatial neglect. In particular, SPOC-M1 connectivity in the contralesional hemisphere was higher in post-stroke neglect patients (and particularly those presenting with peripersonal neglect). This finding provides potentially important insight into the contribution of interhemispheric rivalry to the physiopathology of neglect, which may be correlated with the functional status of connections between the most caudal sites in the PPC and motor areas. In the contrary, motor impairment did not seem to depend on PPC-M1 connectivity at rest.

\section{References}

Azouvi P, Olivier S, de Montety G, Samuel C, Louis-Dreyfus A, Tesio L. Behavioral assessment of unilateral neglect: study of the psychometric properties of the Catherine Bergego Scale. Arch Phys Med Rehabil 2003;84:51-7.

Baas U, de Haan B, Grässli T, Karnath H-O, Mueri R, Perrig WJ, et al. Personal neglect-a disorder of body representation? Neuropsychologia 2011;49:898-905.

Baldassarre A, Ramsey L, Hacker CL, Callejas A, Astafiev SV, Metcalf NV, et al. Large-scale changes in network interactions as a physiological signature of spatial neglect. Brain J Neurol 2014; 137:3267-83.

Bozzali M, Mastropasqua C, Cercignani M, Giulietti G, Bonnì S, Caltagirone C, et al. Microstructural damage of the posterior corpus callosum contributes to the clinical severity of neglect. PloS One 2012;7:e48079.

Buneo CA, Andersen RA. The posterior parietal cortex: Sensorimotor interface for the planning and online control of visually guided movements. Neuropsychologia 2006;44:2594-606.

Busan P, Barbera C, Semenic M, Monti F, Pizzolato G, Pelamatti G, et al. Effect of Transcranial Magnetic Stimulation (TMS) on Parietal and Premotor Cortex during Planning of Reaching Movements. Lu B, editor. PLoS ONE 2009;27:e4621.

Cavina-Pratesi C, Monaco S, Fattori P, Galletti C, McAdam TD, Quinlan DJ, et al. Functional Magnetic Resonance Imaging Reveals the Neural Substrates of Arm Transport and Grip 
Formation in Reach-to-Grasp Actions in Humans. J Neurosci 2010;30:10306-23.

Cocchini G, Beschin N, Jehkonen M. The Fluff Test: A simple task to assess body representation neglect. Neuropsychol Rehabil 2001;11:17-31.

Corbetta M, Shulman GL. Control of goal-directed and stimulus-driven attention in the brain. Nat Rev Neurosci 2002;3:201-15.

Corbetta M, Shulman GL. Spatial neglect and attention networks. Annu Rev Neurosci 2011;34:56999.

Davare M, Andres M, Clerget E, Thonnard J-L, Olivier E. Temporal Dissociation between Hand Shaping and Grip Force Scaling in the Anterior Intraparietal Area. J Neurosci 2007;11;27:397480.

Davare M, Kraskov A, Rothwell JC, Lemon RN. Interactions between areas of the cortical grasping network. Curr Opin Neurobiol 2011;21:565-70.

Davare M, Montague K, Olivier E, Rothwell JC, Lemon RN. Ventral premotor to primary motor cortical interactions during object-driven grasp in humans. Cortex 2009;45:1050-7.

Di Monaco M, Schintu S, Dotta M, Barba S, Tappero R, Gindri P. Severity of unilateral spatial neglect is an independent predictor of functional outcome after acute inpatient rehabilitation in individuals with right hemispheric stroke. Arch Phys Med Rehabil 2011;92:1250-6.

Filimon F. Human cortical control of hand movements: parietofrontal networks for reaching, grasping, and pointing. Neurosci Rev J Bringing Neurobiol Neurol Psychiatry 2010;16:388-407.

Frey SH, Vinton D, Norlund R, Grafton ST. Cortical topography of human anterior intraparietal cortex active during visually guided grasping. Brain Res Cogn Brain Res 2005;23:397-405.

Fugl-Meyer AR, Jääskö L, Leyman I, Olsson S, Steglind S. The post-stroke hemiplegic patient. 1. a method for evaluation of physical performance. Scand J Rehabil Med 1975;7:13-31.

Gauthier L, Dehaut F, Joanette Y. The Bells Test: A quantitative and qualitative test for visual neglect. Int J Clin Neuropsychol 1989;1:49-54.

Heilman KM, Bowers D, Coslett HB, Whelan H, Watson RT. Directional hypokinesia: prolonged reaction times for leftward movements in patients with right hemisphere lesions and neglect. Neurology 1985;35:855-9.

Heilman KM, Valenstein E, Watson RT. Neglect and related disorders. Semin Neurol. 2000;20:46370.

Inman CS, James GA, Hamann S, Rajendra JK, Pagnoni G, Butler AJ. Altered resting-state effective connectivity of fronto-parietal motor control systems on the primary motor network following stroke. NeuroImage 2012;59:227-37.

Jacquin-Courtois S. Hemi-spatial neglect rehabilitation using non-invasive brain stimulation: Or how to modulate the disconnection syndrome? Ann Phys Rehabil Med 2015;58(4):251-8.

Karabanov AN, Chao C-C, Paine R, Hallett M. Mapping Different Intra-Hemispheric Parietal-Motor Networks Using Twin Coil TMS. Brain Stimulat 2013;6:384-9.

Kim E-J, Lee B, Jo M-K, Jung K, You H, Lee BH, et al. Directional and spatial motor intentional disorders in patients with right versus left hemisphere strokes. Neuropsychology 2013;27:42837.

Koch G, Bonnì S, Giacobbe V, Bucchi G, Basile B, Lupo F, et al. $\theta$-burst stimulation of the left hemisphere accelerates recovery of hemispatial neglect. Neurology. 2012;78:24-30.

Koch G, Cercignani M, Bonni S, Giacobbe V, Bucchi G, Versace V, et al. Asymmetry of Parietal Interhemispheric Connections in Humans. J Neurosci 2011;31:8967-75.

Koch G, Cercignani M, Pecchioli C, Versace V, Oliveri M, Caltagirone C, et al. In vivo definition of parieto-motor connections involved in planning of grasping movements. NeuroImage 2010;51:300-12.

Koch G, Del Olmo MF, Cheeran B, Schippling S, Caltagirone C, Driver J, et al. Functional Interplay between Posterior Parietal and Ipsilateral Motor Cortex Revealed by Twin-Coil Transcranial Magnetic Stimulation during Reach Planning toward Contralateral Space. J Neurosci 2008a;28:5944-53.

Koch G, Del Olmo M, Cheeran B, Ruge D, Schippling S, Caltagirone C, et al. Focal Stimulation of the Posterior Parietal Cortex Increases the Excitability of the Ipsilateral Motor Cortex. J Neurosci 2007;27:6815-22.

Koch G, Oliveri M, Cheeran B, Ruge D, Gerfo EL, Salerno S, et al. Hyperexcitability of parietal- 
motor functional connections in the intact left-hemisphere of patients with neglect. Brain 2008b;13:3147-55.

Liepert J, Hamzei F, Weiller C. Motor cortex disinhibition of the unaffected hemisphere after acute stroke. Muscle Nerve 2000;23:1761-3.

Lunven M, Schotten MTD, Bourlon C, Duret C, Migliaccio R, Rode G, et al. White matter lesional predictors of chronic visual neglect: a longitudinal study. Brain 2015;138:746-60.

Makris N, Kennedy DN, McInerney S, Sorensen AG, Wang R, Caviness VS, et al. Segmentation of Subcomponents within the Superior Longitudinal Fascicle in Humans: A Quantitative, In Vivo, DT-MRI Study. Cereb Cortex 2005;15:854-69.

Manganotti P, Patuzzo S, Cortese F, Palermo A, Smania N, Fiaschi A. Motor disinhibition in affected and unaffected hemisphere in the early period of recovery after stroke. Clin Neurophysiol Off $\mathbf{J}$ Int Fed Clin Neurophysiol 2002;113:936-43.

Molenberghs P, Sale MV, Mattingley JB. Is there a critical lesion site for unilateral spatial neglect? A meta-analysis using activation likelihood estimation. Front Hum Neurosci 2012;6:78.

Mort DJ, Malhotra P, Mannan SK, Rorden C, Pambakian A, Kennard C, et al. The anatomy of visual neglect. Brain J Neurol 2003;126:1986-97.

Oldfield RC. The assessment and analysis of handedness: the Edinburgh inventory. Neuropsychologia 1971;9:97-113.

Ptak R, Schnider A. The Dorsal Attention Network Mediates Orienting toward Behaviorally Relevant Stimuli in Spatial Neglect. J Neurosci 2010 ;30:12557-65.

Ptak R, Schnider A. The attention network of the human brain: relating structural damage associated with spatial neglect to functional imaging correlates of spatial attention. Neuropsychologia 2011;49:3063-70.

Ramsey LE, Siegel JS, Baldassarre A, Metcalf NV, Zinn K, Shulman GL, et al. Normalization of network connectivity in hemispatial neglect recovery. Ann Neurol 2016;80:127-41.

Rode G, Cotton F, Revol P, Jacquin-Courtois S, Rossetti Y, Bartolomeo P. Representation and disconnection in imaginal neglect. Neuropsychologia 2010;48:2903-11.

Rossini PM, Barker AT, Berardelli A, Caramia MD, Caruso G, Cracco RQ, et al. Non-invasive electrical and magnetic stimulation of the brain, spinal cord and roots: basic principles and procedures for routine clinical application. Report of an IFCN committee. Electroencephalogr Clin Neurophysiol 1994;91:79-92.

Rothwell JC. Using transcranial magnetic stimulation methods to probe connectivity between motor areas of the brain. Hum Mov Sci 2011;30:906-15.

Rousseaux M, Allart E, Bernati T, Saj A. Anatomical and psychometric relationships of behavioral neglect in daily living. Neuropsychologia 2015;70:64-70.

Rousseaux M, Beis JM, Pradat-Diehl P, Martin Y, Bartolomeo P, Bernati T, et al. [Presenting a battery for assessing spatial neglect. Norms and effects of age, educational level, sex, hand and laterality]. Rev Neurol (Paris) 2001;157:1385-400.

Rousseaux M, Honoré J, Vuilleumier P, Saj A. Neuroanatomy of space, body, and posture perception in patients with right hemisphere stroke. Neurology 2013;81:1291-7.

Schulz R, Buchholz A, Frey BM, Bönstrup M, Cheng B, Thomalla G, et al. Enhanced Effective Connectivity Between Primary Motor Cortex and Intraparietal Sulcus in Well-Recovered Stroke Patients. Stroke 2016;47:482-9

Schulz R, Koch P, Zimerman M, Wessel M, Bönstrup M, Thomalla G, et al. Parietofrontal motor pathways and their association with motor function after stroke. Brain 2015;138:1949-60.

Szczepanski SM, Pinsk MA, Douglas MM, Kastner S, Saalmann YB. Functional and structural architecture of the human dorsal frontoparietal attention network. Proc Natl Acad Sci U S A 2013;110:15806-11.

Uswatte G, Taub E, Morris D, Vignolo M, McCulloch K. Reliability and validity of the upperextremity Motor Activity Log-14 for measuring real-world arm use. Stroke J Cereb Circ 2005;36:2493-6.

Vallar G, Perani D. The anatomy of unilateral neglect after right-hemisphere stroke lesions. A clinical/CT-scan correlation study in man. Neuropsychologia 1986;24:609-22.

Verdon V, Schwartz S, Lovblad K-O, Hauert C-A, Vuilleumier P. Neuroanatomy of hemispatial neglect and its functional components: a study using voxel-based lesion-symptom mapping. 
Brain. 2010;133:880-94.

Vesia M, Bolton DA, Mochizuki G, Staines WR. Human parietal and primary motor cortical interactions are selectively modulated during the transport and grip formation of goal-directed hand actions. Neuropsychologia. 2013;51:410-7.

Vesia M, Prime SL, Yan X, Sergio LE, Crawford JD. Specificity of human parietal saccade and reach regions during transcranial magnetic stimulation. J Neurosci 2010;30:13053-65.

Vossel S, Geng JJ, Fink GR. Dorsal and Ventral Attention Systems. The Neuroscientist. 2014;20:150 9.

Table 1 Characteristics of stroke patients and healthy controls

\begin{tabular}{|c|c|c|c|c|c|c|c|c|}
\hline & Gender & Age (y) & Stroke type & $\begin{array}{c}\text { Median time } \\
\text { since stroke }(\mathbf{m})\end{array}$ & $\begin{array}{c}\text { FMA-UE } \\
\text { score (out of } \\
66 \text { ) }\end{array}$ & $\begin{array}{l}\text { Bells test (total } \\
\text { omissions) }\end{array}$ & $\begin{array}{l}\text { Fluff test } \\
\text { (left score, } \\
\text { out of 15) }\end{array}$ & $\begin{array}{l}\text { CBS score } \\
\text { (out of 30) }\end{array}$ \\
\hline Patients & $\begin{array}{l}M=10 \\
(83 \%)\end{array}$ & $\begin{array}{c}53.1 \pm 14 \\
2\end{array}$ & $\mathrm{I}=9 \quad(75 \%)$ & 15 (142) & $33.7 \pm 20.4$ & & & \\
\hline \multirow[t]{7}{*}{$N+$ group } & $\begin{array}{l}M=4 \\
(66 \%)\end{array}$ & $60 \pm 8.1$ & $\mathrm{I}=5(83.3 \%)$ & $12(91)$ & $26 \pm 22.9$ & & & \\
\hline & $M$ & 54 & $I$ & 17 & 11 & 8 & 14 & 7 \\
\hline & $F$ & 64 & $I$ & 7 & 24 & 7 & 10 & 11 \\
\hline & $M$ & 58 & $I$ & 6 & 60 & 4 & 12 & 7 \\
\hline & $M$ & 69 & $I$ & 186 & 6 & 6 & 15 & 4 \\
\hline & $M$ & 67 & $H$ & 6 & 7 & 9 & 13 & 9 \\
\hline & $F$ & 48 & $I$ & 66 & 48 & 6 & 14 & 10 \\
\hline \multirow[t]{7}{*}{$N$-group } & $\begin{array}{c}M=6 \\
(100 \%)\end{array}$ & $46 \pm 16.1$ & $\mathrm{I}=4(66,6 \%)$ & $56(164)$ & $41 \pm 15.7$ & & & \\
\hline & $M$ & 40 & $H$ & 11 & 33 & - & - & - \\
\hline & $M$ & 54 & $I$ & 13 & 59 & - & - & - \\
\hline & $M$ & 55 & $I$ & 99 & 38 & - & - & - \\
\hline & $M$ & 67 & $I$ & 202 & 33 & - & - & - \\
\hline & $M$ & 20 & $I$ & 164 & 23 & - & - & - \\
\hline & $M$ & 41 & $H$ & 6 & 62 & - & - & - \\
\hline Controls & $\begin{array}{l}M=9 \\
(75 \%)\end{array}$ & $51 \pm 10.9$ & - & - & - & - & - & - \\
\hline
\end{tabular}

Stroke type: I=ischemic; H=hemorrhagic; FMA-UE: upper extremity subsection of the Fugl-Meyer Assessment; CBS: 


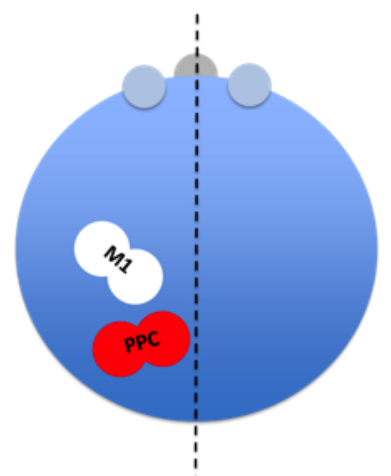

b

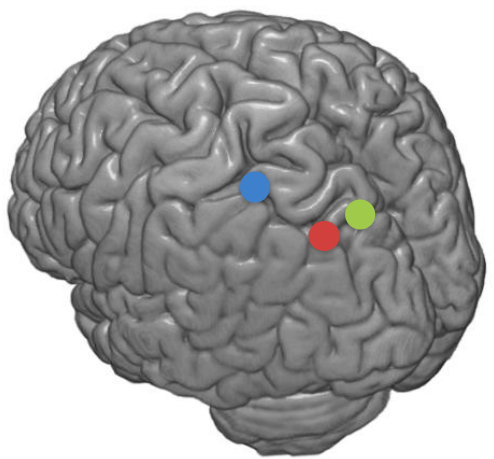

Fig 1. (a) Schematic illustration of the coil positioning in our ppTMS experiment. The red ellipses represent the coil delivering the CS over each of the 3 PPC regions, and the white ellipses represent the coil delivering the TS over M1. (b) Stimulation sites over the aIPS (blue), pIPS (red) and SPOC (green) in the left hemisphere.

ALL PATIENTS

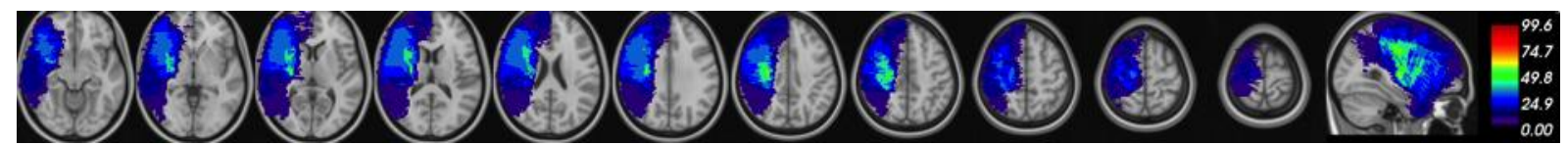

N+ PATIENTS

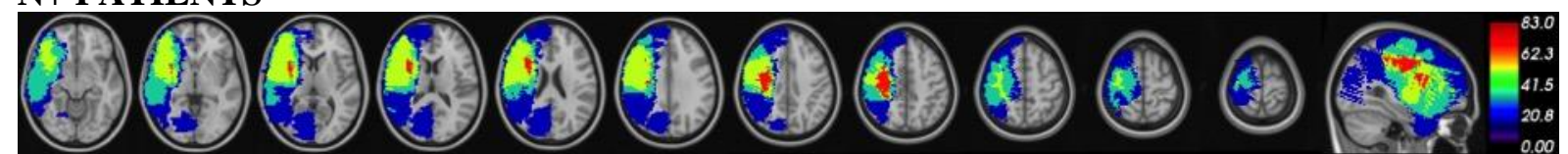

N- PATIENTS

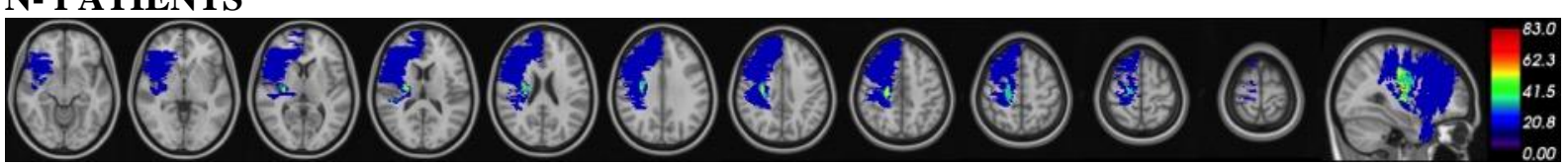


Fig. 2 Lesion overlap map for the group of patients as a whole (ALL PATIENTS), patients with neglect (N+ PATIENTS) and patients without neglect (N-PATIENTS). The color scale at the end of each row indicates the proportion of patients with a lesion in that voxel (higher for colors further towards the top of the scale).

a

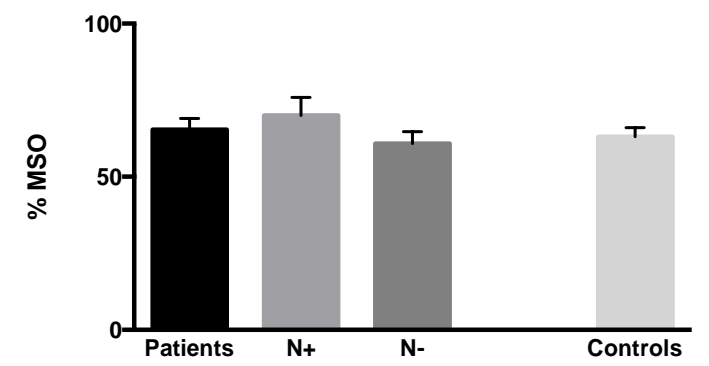

b

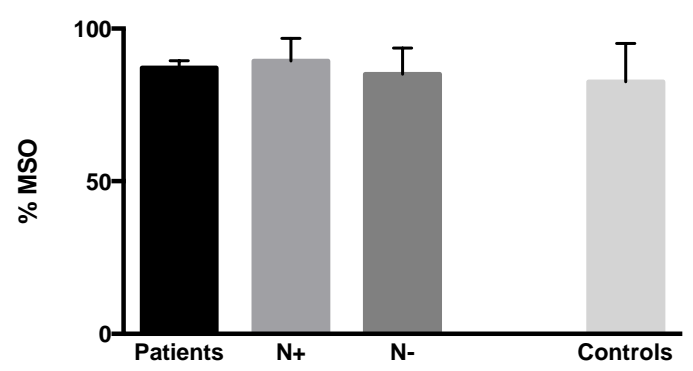

Fig. 3. The RMT in the left (contralesional) motor cortex (a) and the intensity of the TS over the left M1 needed to elicit a $1 \mathrm{mV}$ MEP (b) in the different study groups (all patients; patients with neglect $(\mathrm{N}+$ ), patients without neglect (N-), and healthy controls), as a percentage of the maximum stimulator output. Errors bars correspond to the SEM. 


\section{aIPS}

a

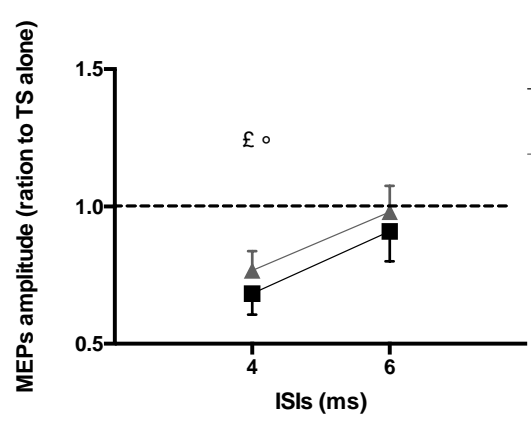

C

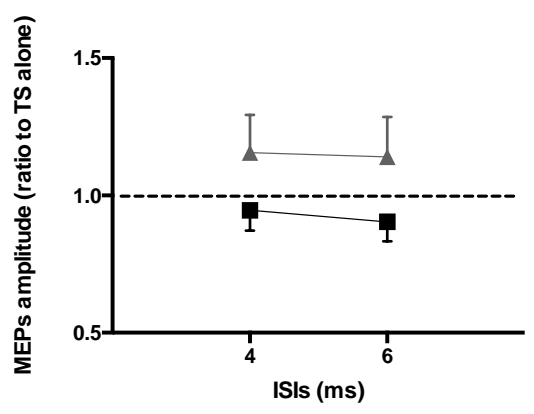

e

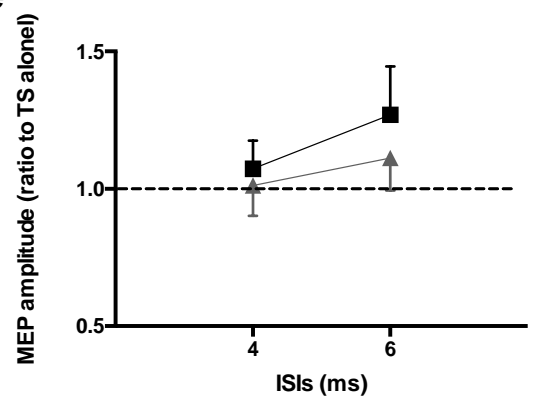

b

Patients $(n=8)$

Controls $(n=10)$ $\diamond N+(n=5)$

- $N-(n=5)$
pIPS

d

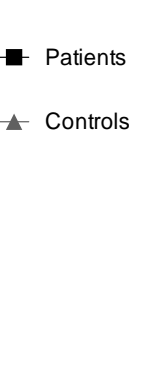

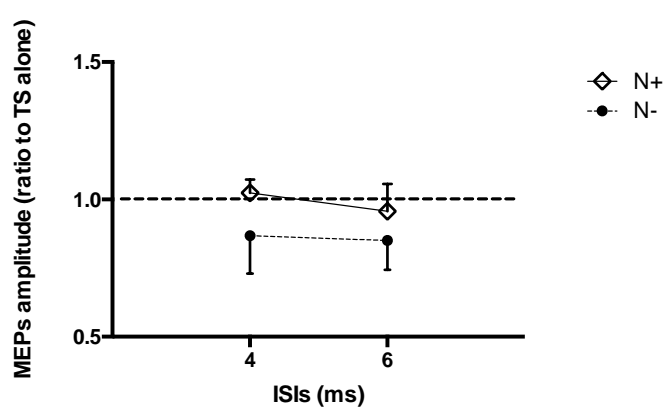

SPOC

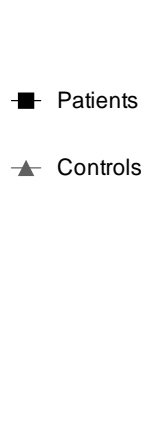

f

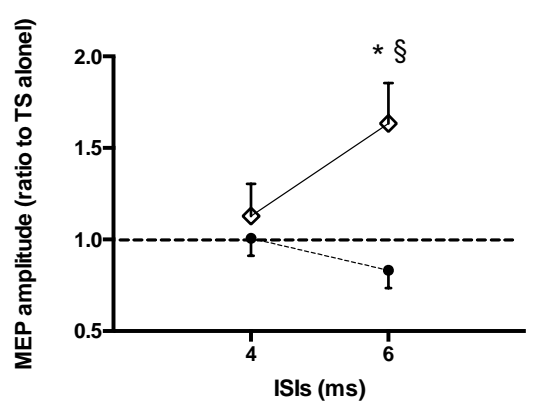

Fig. 4 PPC-M1 connectivity for each of the two ISIs when the CS

was applied over the aIPS (a, b), pIPS (c, d) or SPOC (e,f). Graphs in the left-hand column display data from all patients vs. healthy controls, whereas graphs in the right-hand column display data from patients with neglect $(\mathrm{N}+)$ vs. those without neglect (N-). For each condition, the MEP amplitude was normalized against TS alone (the dotted line represents the TS condition). The protocol was not performed for the aIPS in 2 patients (one in each group) and in 4 healthy controls.

Intergroup differences: ${ }^{\S} \mathrm{p}<0.05$ vs. the $\mathrm{N}$ - group

Intragroup differences: ${ }^{£} \mathrm{p}<0.05$ vs. the control condition (TS alone) in patients; ${ }^{\circ} \mathrm{p}<0.05$ vs. the control condition in healthy controls $* \mathrm{p}<0.05$ vs. control condition in the $\mathrm{N}+$ group; Error bars correspond to the SEM. 


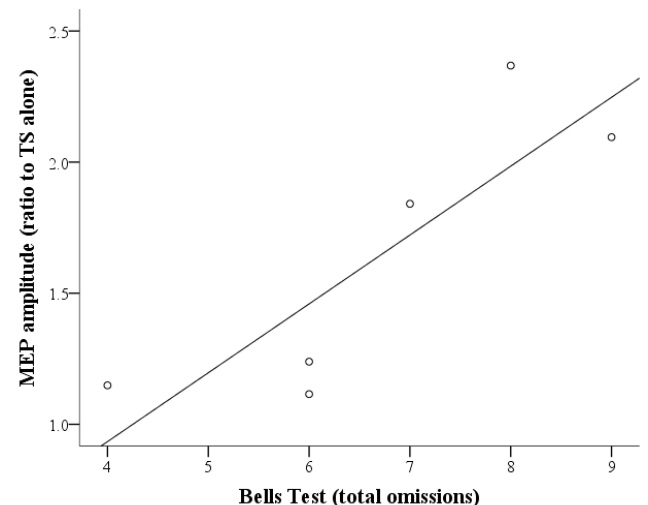

Fig 5. MEP facilitation as a function of the severity of peripersonal spatial neglect, as assessed by the total number of Bells test omissions in the 6 patients in the $\mathrm{N}+$ group. 